\title{
In-vehicle UWB Channel Measurement, Model and Spatial Stationarity
}

\author{
Jiri Blumenstein, Tomas Mikulasek, \\ Roman Marsalek, Aniruddha Chandra, Ales Prokes \\ The Faculty of Electrical Engineering and Communication \\ Brno University of Technology \\ Brno, Czech Republic \\ Email: blumenstein@feec.vutbr.cz
}

\author{
Thomas Zemen
}

FTW Forschungszentrum Telekommunikation Wien, Vienna University of Technology, Vienna, Austria

\author{
Christoph Mecklenbräuker \\ Institute of Telecommunications, \\ Vienna, Austria
}

\begin{abstract}
This contribution documents an ultra-wide band (UWB) channel measurement performed in an in-vehicle environment for the frequency range 3-11 GHz.

An emphasis is placed into an evaluation of a spatial consistency of measured channel characteristics in terms of Pearson correlation between measured channel impulse responses (CIRs). Moreover, the measured CIRs are reproducible via a two-part exponentially decaying envelope-delay profile (EDP). The small scale variation of received signal is parametrized utilizing a random process obeying the generalized extreme value (GEV) distribution.
\end{abstract}

Validation of the channel model is demonstrated utilizing a two sample Kolmogorov-Smirnov (K-S) test.

\section{INTRODUCTION}

The effort for the efficient personal mobility is unimaginable without wireless connectivity between vehicles, roadside infrastructure and also inside of each individual vehicle. UWB technology, with its $10 \mathrm{GHz}$ of unlicensed bandwidth [1] and beneficial short-range multipath characteristics [2], [3], can act as wireless hub for connecting wireless personal devices of the passengers with the vehicle.

Considering the average weight of wire harness in modern vehicles exceeding several tents of kilograms [4], the UWB technology can also be exploited as a wireless bus supporting non-critical safety functionalities of the vehicle. This approach will also lower the wire harness design dependency on a specific vehicle platform thus enhances universality of the vehicle's communication systems. Connection of moving parts, such as wheels for tyre pressure monitoring [5], or the rear seat entertainment system is also beneficially realized wirelessly.

The UWB single-input single-output (SISO) link is examined in [6], [7] considering a frequency range $3-8 \mathrm{GHz}$ and without a spatial correlation study. In [8], [9], the spatial distribution of path gains in the vertical and horizontal plane as well as power-delay profiles are presented, however no channel model for a reproduction of the measured data is provided.

\section{A. Contribution of the Paper}

- This paper presents results of an in-vehicle multiple input single output (MISO) channel measurement campaign performed in the 3-11 GHz frequency band.
- We study the spatial channel stationarity evaluated via Pearson correlation coefficients between absolute values of measured CIRs.

- The exponentially decaying EDP channel model validated with the two-sample K-S test [10].

The rest of the paper is organized as follows: Firstly we provide a measurement setup description, secondly the correlation coefficients measurement is presented, and then we present our channel model and its validation. Conclusion rounds up the paper.

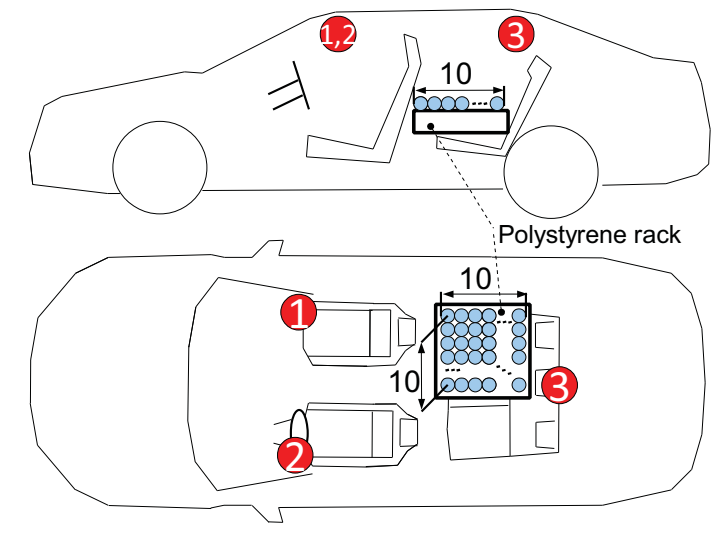

Fig. 1. Antennas layout, RED-three transmitting antennas, BLUE-receiving antenna. We utilized one receiving antenna, which has been manually moved over the depicted polystyrene grid ensuring defined antenna positions. The grid distance in both directions is $3 \mathrm{~cm}$, the grid allows to measure $10 \times 10$ positions.

\section{MeAsurement SETUP}

Measurements are performed in a mid-sized passenger car Skoda Octavia III with transmit and receive antennas marked with red and blue colors in Figure 1 respectively. The transmitting (TX) antennas are placed on the two top corners of the front windshield and at the rear part of the ceiling according to Figure 1 and Figure 2. The receiving antenna is placed at $10 \times 10$ spatial points using the polystyrene rack which ensured defined location of the receiving antenna. The polystyrene rack utilizes a $3 \mathrm{~cm}$ grid distance between neighboring measurement locations. 

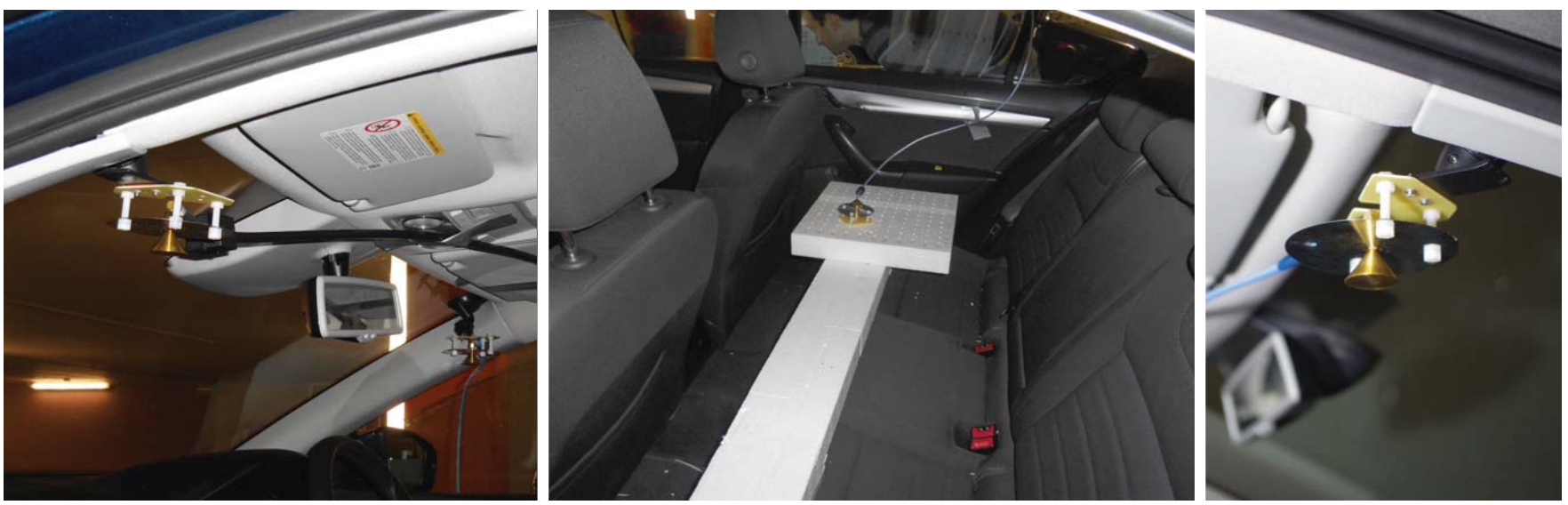

Fig. 2. From left to right: Two transmitting omni-directional conical monopole antennas mounted on the top corners of the front windshield; $10 \times 10$ polystyrene rack for defined placement of the receiving antenna on the rear seat; Detail of the conical monopole antenna. (Note: The rear transmitting antenna mounted on the ceiling is not depicted)

The 4-port vector network analyzer Agilent Technologies E5071C (VNA) is used for measuring the transmission coefficient between two antennas in the frequency band 3-11 $\mathrm{GHz}$. The omni-directional conical monopole antennas having the radiation pattern depicted in Figure 3 are exploited as a transmitting and receiving antennas.

Note that the measurement is performed in the frequency domain with $100 \mathrm{MHz}$ resolution bandwidth. Then we transform the channel frequency response into the time domain utilizing the fast Fourier Transform (FFT) with rectangular window.
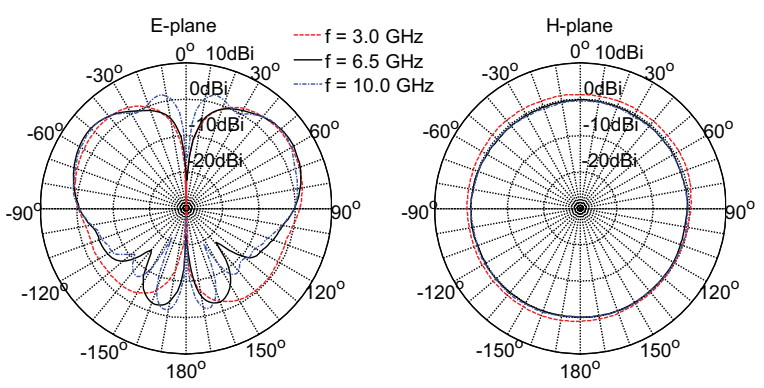

Fig. 3. Simulated gain pattern of the conical monopole antennas.

\section{CHANNEL DESCRIPTION}

\section{A. Channel Impulse Response (CIR)}

The intra vehicle ambiance is described by its impulse response:

$$
h_{\alpha}^{\prime k}(\tau)=\sum_{n=0}^{N-1} \gamma_{n}^{\alpha} \mathrm{e}^{j \Phi_{n}^{\alpha}} \delta\left(\tau-\tau_{n}\right),
$$

where $\tau_{n}$ is the propagation delay and $\gamma_{n}^{\alpha} \mathrm{e}^{j \Phi_{n}^{\alpha}}$ is the complex amplitude coefficient of the $n$-th multipath component belonging to $\alpha$-th wireless link. Since three transmitting antennas are utilized in this measurement campaign, the index $\alpha$ marks individual wireless links between the transmitting antennas

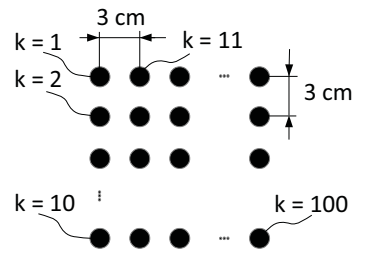

Fig. 4. Layout of the receiving antenna. Each specific point is marked with $k$ according to depicted logic. The distance between grid neighboring positions is $3 \mathrm{~cm}$.

and the receiving antenna. The superscript $k$ denotes the position of the receiving antenna. As evident from Figure 1, we have exploited the $10 \times 10$ grid which allows us to use $k \in\{1 \ldots 100\}$.

The $h_{\alpha}^{\prime k}(\tau)$ is a complex signal with uniformly distributed phase in the interval $[0,2 \pi)$. In the following text, we use the absolute value of the CIR, $h_{\alpha}^{k}(\tau)=\left|h_{\alpha}^{\prime k}(\tau)\right|$. Note that the in-vehicle channel is considered as time invariant.

\section{B. Spatial Stationarity of Channel Impulse Response}

The spatial stationarity of the measured wireless channel is examined via the evaluation of the Pearson correlation coefficient $\rho_{\alpha}^{k}$ of the measured CIR $h_{\alpha}^{k}(\tau)$ which is given as:

$$
\rho_{\alpha}^{k}=\frac{E\left[\left(h_{\alpha}^{k}(\tau)-\mu_{\alpha}^{k}\right)\left(h_{\alpha}^{45}(\tau)-\mu_{\alpha}^{45}\right)\right]}{\sigma_{\alpha}^{k} \sigma_{\alpha}^{45}},
$$

where $\sigma_{\alpha}^{k}$ denotes the variance and $\mu_{\alpha}^{k}$ the mean of $h_{\alpha}^{k}(\tau)$. The operator $E[-]$ denotes the expected value. The correlations are calculated between positions $k \in\{1 \ldots 100\}$ and $k=45$ which is located approximately in the middle of the grid.

The results are plotted in Figure 5. Reflecting the square geometry of our polystyrene rack, the map of correlation coefficient $\rho_{1}^{k}$ shows that $h_{1}^{k}(\tau)$ is spatially stationary with a mean value of $\rho_{1} \approx 0.55$. The coefficients $\rho_{1}^{k}$ slightly decrease with the distance form the reference point $k=45$. This behavior is typical also for $\rho_{2,3}$. The histograms of $\rho_{1,2,3}$ are visible in Figure 5. Let us note that for the following processing we take into account only the CIRs with $\rho_{\alpha}>0.5$. 

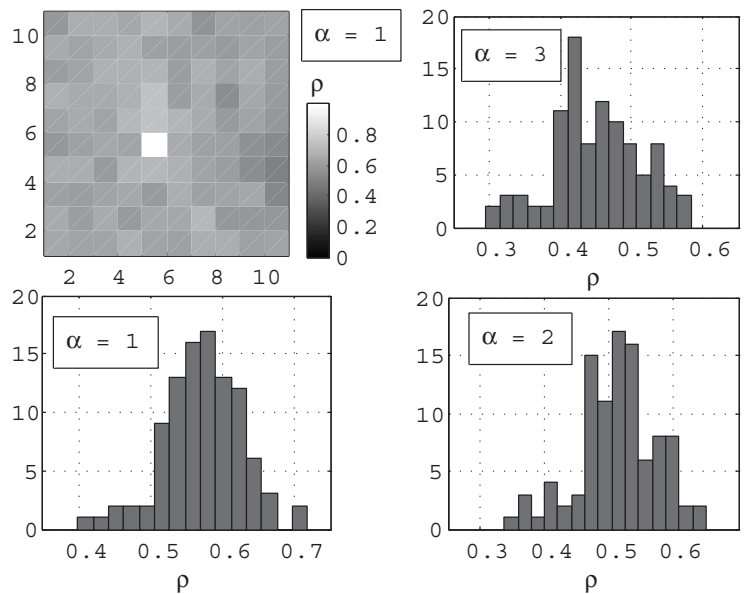

Fig. 5. From top left corner: The map of Pearson correlation coefficient $\rho_{1}$ for all spatial point $k$ taking into account the square geometry of our rack; Histograms of $\rho_{\alpha}$ for $\alpha \in\{1 \ldots 3\}$. Let us note that for the following processing we take into account only the CIRs with $\rho_{\alpha}>0.5$.

\section{Channel Model}

Let us assume that the CIR is composed of large-scale variations (LSV) and small-scale variations (SSV) according to: $h_{\alpha}(\tau)=\wp_{\alpha}(\tau)+\xi_{\alpha}(\tau)$, where $\wp_{\alpha}(\tau)$ and $\xi_{\alpha}(\tau)$ stands for the LSV and SSV respectively. According to our observations and with the aim to provide a reproducible channel model, we operate with the hypothesis that the SSV is simulated with an appropriate random process while the LSV phenomena is described by a two-part exponentially decaying EDP function as: $\wp_{1}^{*}(\tau)=\wp_{1}^{1 *}(\tau)+\wp_{1}^{2 *}(\tau)$ describing the direct/dominant component (DC) and reverberant diffuse tail (RDT) as depicted in [11]. Note that $*$ marks modeled variables.

This approach is enabled by the possibility to decompose the CIR into LSV and SSV by averaging the CIR over spatially correlated area according to $\operatorname{EDP}_{\alpha}(\tau)=E\left[\left|h_{\alpha}^{\prime k}(\tau)\right|\right]$.

As visible in Figure 6, where the comparison of measured CIRs $h_{1}^{k} \forall \rho_{k}>0.5$, spatial average $\mathrm{EDP}_{1}$ and accordingly derived LSV model $\wp_{1}^{1 *}$ and $\wp_{1}^{2 *}$ is seen, the EDP still contains residual influence of the SSV. Therefore, the exploitation of the Hodrick-Prescott (H-P) detrending filter [12] which operates with so called cyclical and trend components is proposed in order to get rid of the residual SSV. If not used, the channel model would not pass the K-S test. A similar method is discussed in [13].

\section{A. Large Scale Variations}

For a randomly selected index $k$, the $h_{\alpha}^{k}(\tau)$ is filtered with the H-P de-trending filter according to (3), the cyclical component $\xi_{\alpha}(\tau)$ is then acquired [12] as:

$$
\xi_{\alpha}(\tau)=\min _{\wp}\left(\sum_{\tau=1}^{T}\left(h_{\alpha}^{k}(\tau)-\wp_{\alpha}(\tau)\right)^{2}+\right.
$$

$$
\left.+\lambda \sum_{\tau=2}^{T-1}\left[\left(\wp_{\alpha}(\tau+1)-\wp_{\alpha}(\tau)\right)-\left(\wp_{\alpha}(\tau)-\wp_{\alpha}(\tau-1)\right)\right]^{2}\right),
$$
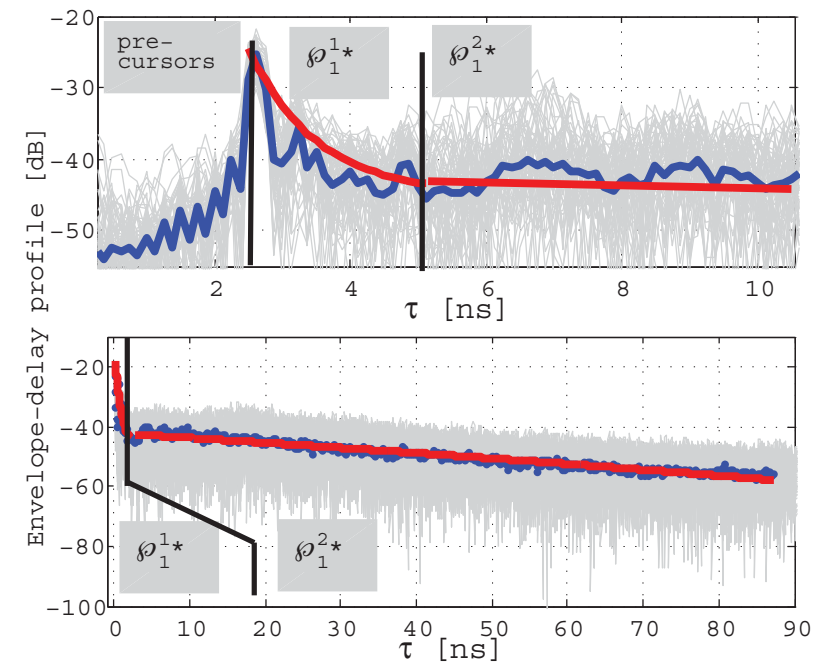

Fig. 6. [Top] Detail of measured $h_{1}^{k} \forall \rho_{1}^{k}>0.5$ (gray), spatial average $\mathrm{EDP}_{1}$ (blue) and accordingly derived EDP model $\wp_{1}^{1 *}$ and $\wp_{1}^{2 *}$ (red) representing the DC and RDT respectively. [bottom] The whole perspective of $\wp_{1}^{1 *}$ with a time window of $4 \mathrm{~ns}$ and the $\wp_{1}^{2 *}$ with duration of $80 \mathrm{~ns}$.

where $\wp_{\alpha}$ is a trend component and $\tau \in\{1, \ldots, T\}$ denotes time in the delay domain. Let us note that according to the terminology utilized in [12] we consider the cyclical component to represent the SSV and the LSV is modeled by the trend component.

The heuristically chosen multiplier $\lambda$ adapts the filter in order to filter out the LSV. In this paper we use $\lambda=500$, nevertheless the set of possible values of $\lambda$ ranges from $\lambda=10$ up to $\lambda=500$, while the lower the $\lambda$, the faster the $\wp$ changes. We have chosen the $\lambda$ in the upper range of possible values in order to provide the curve fit of the LSV of the lowest possible order while still maintaining the two-sample K-S $p$-values in the $>0.1$ region ensuring "very significant match" according to the ingrained interpretation of two-sample K-S test results. More information regarding the adjustment of $\mathrm{H}-\mathrm{P}$ filter is elaborated in [14].

When compared with a moving average filter, the H-P does not cause data loss, thus seems beneficial. In general however, application of other filters is possible.

Next, to obtain the LSV component $\wp_{\alpha}(\tau)$, the cyclical component $\xi_{\alpha}(\tau)$ is subtracted from the measured CIR, $\wp_{\alpha}(\tau)=h_{\alpha}^{k}(\tau)-\xi_{\alpha}(\tau)$. Note that we take into consideration only data starting from the first multipath component $\gamma_{1}^{\alpha}$.

To model the measured LSV phenomena, we parametrize the $\wp_{\alpha}(\tau)$ utilizing two-part exponentially decaying function pursuant to:

$$
\begin{gathered}
\wp_{\alpha}^{1 *}(\tau)=A_{\alpha}+10 B_{\alpha} \exp \left(-C_{\alpha} \tau\right), \forall \tau \in\{0 \ldots 4 \mathrm{~ns}\}, \\
\wp_{\alpha}^{2 *}(\tau)=D_{\alpha} \exp \left(E_{\alpha} \tau\right), \forall \tau \in\{4 \ldots 85 \mathrm{~ns}\},
\end{gathered}
$$

with parameters listed in Table I.

\section{B. Small Scale Variations}

Exploiting the maximum likelihood estimation (MLE), we parametrize the superimposed SSV signal $\xi_{\alpha}(\tau)$ using the 
TABLE I

PARAMETERS OF TWO-PIECE EXPONENTIAL APPROXIMATION OF EDP MODEL $\wp_{\alpha}^{1 *}, \wp_{\alpha}^{2 *}$ AND SSV MODEL $\xi^{*}$

\begin{tabular}{l||l|l||l} 
& $\wp_{\alpha}^{1}$ & $\wp_{\alpha}^{2}$ & $\xi^{*}(\tau)$ \\
\hline \hline$\alpha=1$ & $A_{1}=-45.760$ & $D_{1}=-41.490$ & $\mathrm{~K}=-0.1439$ \\
& $B_{1}=1.420$ & $E_{1}=0.003$ & $\Upsilon=5.9778$ \\
& $C_{1}=0.830$ & & $\Gamma=-2.8511$ \\
\hline$\alpha=2$ & $A_{2}=665.600$ & $D_{2}=-42.410$ & \\
& $B_{2}=-665.900$ & $E_{2}=0.003$ & \\
& $C_{2}=-0.7 \mathrm{e}-3$ & & \\
\hline$\alpha=3$ & $A_{3}=55.260$ & $D_{3}=-42.290$ & \\
& $B_{3}=3.800$ & $E_{3}=3 \mathrm{e}-3$ & \\
& $C_{3}=0.7 \mathrm{e}-3$ & &
\end{tabular}

generalized extreme value (GEV) distribution [15]. The probability density function (PDF) of the GEV is given by:

$f(x \mid K, \Upsilon, \Gamma)=\frac{1}{\Gamma} \exp \left[-\beta^{-\frac{1}{K}}\right] \beta^{-1-\frac{1}{K}}, \forall \beta=1+K \frac{x-\Upsilon}{\Gamma}$,

where $K$ is the shape parameter, $\Upsilon$ is the scale parameter and $\Gamma$ the distribution location parameter. Presented in Table I, the parameters are computed as a mean value $\xi^{*}=E\left[\xi_{\alpha}\right]$ for all wireless links $\alpha$ making the SSV model spatially independent.

Due to a high flexibility and spatial universality of the SSV model $\xi^{*}$, which is given by three input parameters as opposed to usual two parameters, the MLE metric recommends the GEV distribution. On other hand, authors in [16] claim that there is no theoretical explanation for encountering this distribution type. We may however add, that the GEV contains the accepted log-Weibull distribution as a special case for $K=0$.

\section{Validation of the Channel Model}

To validate our in-vehicle channel model, we have performed the two-sample K-S test. We have compared all $h_{\alpha}^{k}$ with corresponding sum of $\wp_{\alpha}^{1 *}+\wp_{\alpha}^{2 *}+\xi^{*}$ models while resulting $p$-values are visible in Figure 7. Most of the $p$-values for $\alpha=1$ and more then two third of calculated $p$-values for $\alpha=2$ are in $>0.1$ region. Thus, according to ingrained interpretation of $\mathrm{K}-\mathrm{S}$ test, computed $p$-values reports very significant match of our modeling approach. The model is less reliable for $\alpha=3$. Also, as visible in Figure 5, the spatial correlations are significantly lower. This is probably due to the radiation pattern of the conical monopole antennas showing weak radiation in the direction of link $\alpha=3$.

\section{CONCLUSION}

This paper presents reproducible results of in-vehicle MISO UWB channel measurement campaign. Moreover, we evaluate the Pearson correlations of CIRs over a specified area.

We have decomposed the CIR into large-scale variations (LSV) and small-scale variations (SSV) by averaging the spatially correlated CIRs. The resulting envelope-delay profile was moreover deprived of residual SSV influence using Hodrick-Prescott detrending filter. The measured LSV was then approximated utilizing the two-part exponentially decaying function. The SSV is parametrized with spatially invariable generalized extreme value distributed random process.

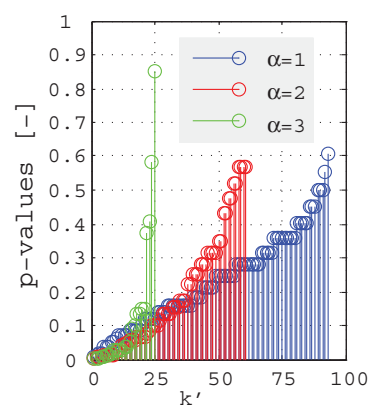

Fig. 7. The spatial positions $k^{\prime} \forall \rho>0.5$ are sorted to plot the respective $p$-values in ascending order comparing the measured CIRs with exponentially decaying EDP model $\wp_{\alpha}^{1 *}$ and $\wp_{\alpha}^{2 *}$ of LSV (input parameters according to Table I) with superimposed GEV distributed SSV model $\xi^{*}(-0.1439,2.8511,5.9778)$.

\section{ACKNOWLEDGMENT}

This work was supported by the Czech Science Foundation project No. 1338735S Research into wireless channels for intra-vehicle communication and positioning, from the SoMoPro II Programme, co-financed by the European Union and the South-Moravian Region and was performed in laboratories supported by the SIX project, No. CZ.1.05/2.1.00/03.0072, the operational program Research and Development for Innovation. The cooperation in the COST IC1004 action was supported by the MEYS of the Czech Republic project no. LD12006 (CEEC). The Christian Doppler Laboratory for Wireless Technologies for Sustainable Mobility is also gratefully acknowledged.

\section{REFERENCES}

[1] Federal Communications Commission, "First report and order, revision of part 15 of commissions rule regarding uwb transmission system FCC 02-48," Washington, DC, April, vol. 22, 2002.

[2] M. Win and R. Scholtz, "Characterization of ultra-wide bandwidth wireless indoor channels: a communication-theoretic view," Selected Areas in Communications, IEEE Journal on, vol. 20, no. 9, 2002.

[3] R.-M. Cramer, R. Scholtz, and M. Win, "Evaluation of an ultra-wideband propagation channel," Antennas and Propagation, IEEE Transactions on, vol. 50, no. 5, pp. 561-570, 2002.

[4] G. Leen and D. Heffernan, "Expanding automotive electronic systems," Computer, vol. 35, no. 1, pp. 88-93, 2002.

[5] G. Lasser and C. Mecklenbrauker, "Channel model for tyre pressure monitoring systems (TPMS)," in Antennas and Propagation (EuCAP), 2010 Proceedings of the Fourth European Conference on. IEEE, 2010

[6] M. Schack, J. Jemai, R. Piesiewicz, R. Geise, I. Schmidt, and T. Kurner, "Measurements and analysis of an in-car UWB channel," in Vehicular Technology Conference, 2008. VTC Spring 2008. IEEE, 2008.

[7] M. Schack, R. Geise, I. Schmidt, R. Piesiewiczk, and T. Kurner, "UWB channel measurements inside different car types," in Antennas and Propagation, 2009. EuCAP 2009. 3rd European Conference on, 2009.

[8] T. Tsuboi, J. Yamada, and N. Yamauchi, "UWB radio propagation inside vehicle environments," in Telecommunications, 2007. ITST '07. 7th International Conference on ITS, 2007, pp. 1-5.

[9] T. Kobayashi, "Measurements and characterization of ultra wideband propagation channels in a passenger-car compartment," in Spread Spectrum Techniques and Applications, 2006 IEEE Ninth International Symposium on, 2006, pp. 228-232.

[10] F. J. Massey Jr, "The Kolmogorov-Smirnov test for goodness of fit," Journal of the American statistical Association, vol. 46, no. 253, pp. 68-78, 1951.

[11] T. Pedersen, G. Steinbock, and B. Fleury, "Modeling of reverberant radio channels using propagation graphs," Antennas and Propagation, IEEE Transactions on, vol. 60, no. 12, pp. 5978-5988, Dec 2012.

[12] R. J. Hodrick and E. C. Prescott, "Postwar US business cycles: an empirical investigation," Journal of Money, credit, and Banking, 1997.

[13] J. Blumenstein, T. Mikulasek, R. Marsalek, A. Prokes, T. Zemen, and C. Mecklenbrauker, "In-vehicle mm-wave channel model and measurement (accepted for publication)," in Vehicular Technology Conference (VTC Fall), 2014 IEEE 80th. IEEE, 2014, pp. 1-5.

[14] M. O. Ravn and H. Uhlig, "On adjusting the Hodrick-Prescott filter for the frequency of observations," Review of economics and statistics, vol. 84, no. 2, pp. 371-376, 2002.

[15] S. Kotz and S. Nadarajah, Extreme value distributions. World Scientific, 2000 .

[16] H. Hashemi, "The indoor radio propagation channel," Proceedings of the IEEE, vol. 81, no. 7, pp. 943-968, 1993. 